\title{
Vancomycin-induced Linear IgA Bullous Dermatosis: A Case Report and Review of the Literature
}

\author{
Min Ju Kang, M.D., Hyung Ok Kim, M.D., Young Min Park, M.D. \\ Department of Dermatology, The Catholic University of Korea, \\ Kangnam St. Mary's Hospital, Seoul, Korea
}

\begin{abstract}
Linear IgA bullous dermatosis (LABD) is a rare autoimmune bullous disease that can either occur without any apparent cause or be induced by the administration of certain drugs, the most common of which is vancomycin. We present a case of a 45 -year-old woman who was diagnosed with vancomycin-induced LABD by the presence of a characteristic linear band of IgA along the basement membrane zone on direct immunofluorescence microscopy. Our patient showed complete recovery after a 2 -week period during which vancomycin administration was discontinued.
\end{abstract}

(Ann Dermatol (Seoul) 20(2) 102 106, 2008)

Key Words: Linear IgA bullous dermatosis, Vancomycin

\section{INTRODUCTION}

Linear IgA bullous dermatosis (LABD) is a rare acquired autoimmune skin disorder that presents as subepidermal blisters. It can be diagnosed by confirming the presence of a linear band of IgA along the basement membrane zone on direct immunofluorescence microscopy ${ }^{1}$. Although LABD is usually idiopathic, it may be induced by drugs, malignancies, or infections. Many drugs, such as acetaminophen', amiodarone $^{3}$, furosemide, and phenytoin, ${ }^{5}$ have been implicated as the cause; however, vancomycin has been found to be the one most commonly associated $^{6}$. To our knowledge, there have been two cases of vancomycin-induced LABD reported in Korea to date. We herein report another case of a 45-year-old woman who presented with vancomycininduced LABD, along with a review of the literature.

Received November 24, 2006

Accepted for publication March 1, 2008

Reprint request to: Young Min Park, M.D., Department of Dermatology, Kangnam St. Mary's Hospital, College of Medicine, The Catholic University of Korea, 505, Banpodong, Seocho-gu, Seoul 137-701, Korea. (Tel) 82-2-5901494, (Fax) 82-2-599-9950,E-mail: yymmpark@hotmail. com

\section{CASE REPORT}

A 45-year-old woman presented with pruritic, multiple, tense vesicles and confluent erythematous papules and plaques on the trunk, inguinal areas, and upper thighs for 3 days (Fig. 1A-C). She had been diagnosed with stomach cancer (stage $\mathrm{T}_{4} \mathrm{~N}_{0} \mathrm{M}_{1}$ ) and accompanying carcinomatosis peritonei and had received palliative total gastrectomy with segmental resection of the transverse colon in May 2004. Afterwards, she underwent 4 cycles of taxoterecisplatin chemotherapy to alleviate some of her symptoms and is currently taking oral 5-fluorouracil as maintenance therapy. Due to the carcinomatosis, both of her ureters were invaded by the cancer cells, which subsequently led to hydronephrosis and persistent urinary tract infection of both kidneys. This was treated by the insertion of double J catheters but the procedure also induced a chronic bacteriuric state.

The patient visited the emergency room in April 2006 with chief complaint of fever and chilling. Laboratory examinations showed a white blood cell count of $14,300 / \mathrm{mm}^{2}$, with $84 \%$ segmented neutrophils, and an elevated blood urea nitrogen and creatinine level $(26.8 \mathrm{mg} / \mathrm{ml} / 3.79 \mathrm{mg} / \mathrm{ml})$. The initial urinalysis showed pyuria and hematuria. This, combined with the patient's symptoms of fever, 

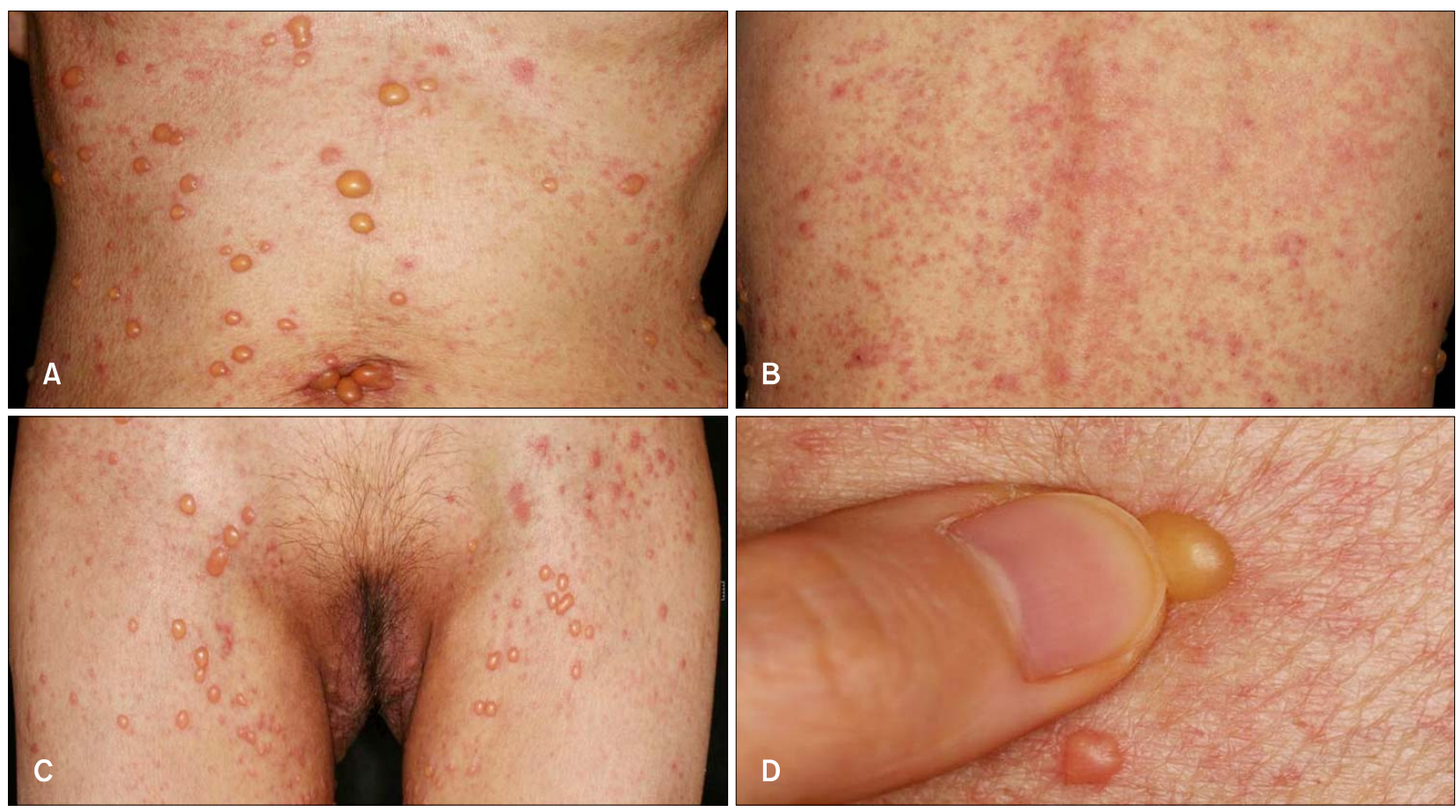

Fig. 1. Pruritic, multiple, tense vesicles and confluent erythematous papules and plaques on the trunk (A, B), inguinal areas, and upper thighs (C). The Asboe-Hansen sign was negative on palpation (D).

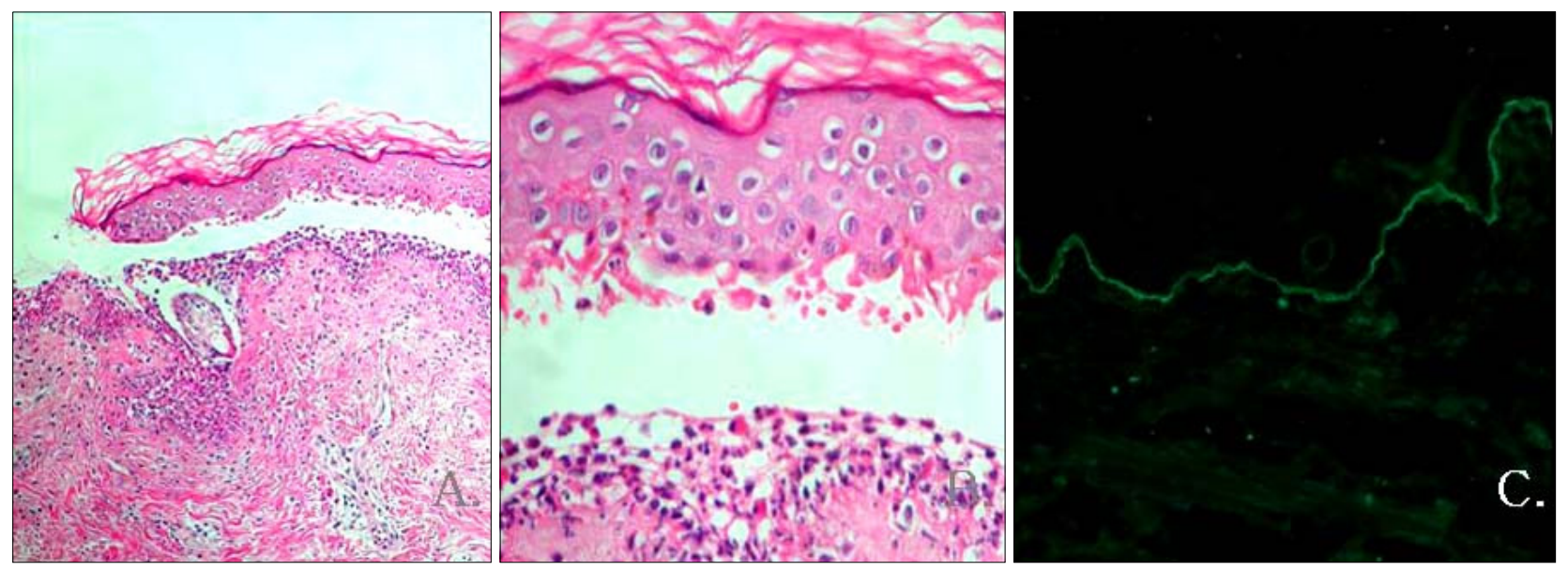

Fig. 2. The biopsy specimen taken from a vesicle on the patient's trunk shows a subepidermal blister, filled with an inflammatory cell infiltrate consisting of neutrophils, a small number of lymphocytes, and eosinophils. Direct immunofluorescence microscopy of the specimen taken from perilesional normal skin demonstrates linear deposits of IgA along the basement membrane zone ( $\mathrm{H} \& \mathrm{E}, \mathrm{A}, \mathrm{C}: \times 100, \mathrm{~B}: \times 400)$.

prompted urine and blood culture. The urine culture showed growth of vancomycin susceptible enterococcus and treatment was initiated with intravenous vancomycin $(2,000 \mathrm{mg} /$ day for 5 days $)$ for the bacteremia. The blood culture revealed growth of
Candida albicans, which was subsequently treated with oral fluconazole (200 mg/day for 14 days). On the 13th day after the initiation of vancomycin, but prior to the fluconazole injection, pruritic, multiple, tense vesicles with an erythematous base and con- 
fluent erythematous papules and plaques suddenly appeared on the trunk, inguinal areas, and upper thighs. An Asboe-Hansen sign was absent (Fig. 1D) and there was no mucosal involvement. Two biopsy specimens were obtained; one from one of the representative vesicles on the trunk, and the other from the perilesional normal skin. The hematoxylin $\&$ eosin stain of the vesicle showed a subepidermal blister, and the blister cavity was filled with inflammatory cell infiltrates, which upon close examination mostly consisted of neutrophils, with a small number of lymphocytes and eosinophils as well (Fig. 2A, B). Direct immunofluorescence microscopy of the specimen taken from perilesional normal skin demonstrated linear deposits of IgA along the basement membrane zone (Fig. 2C). Indirect immunofluorescence microscopy was negative.

Upon the appearance of the skin lesions, vancomycin was singled out as the possible cause, and was subsequently discontinued. After discontinuation, the skin lesions completely resolved over a period of 2 weeks and no recurrence has been reported in the 6 months following resolution. No additional modalities of treatment were required. Based on these clinicopathological findings, the patient was conclusively diagnosed with vancomycin-induced LABD. She was advised not to use vancomycin again in the future to prevent further recurrences.

\section{DISCUSSION}

Although drug-induced LABD is uncommon, its incidence has been steadily increasing in frequency in recent years. Several drugs have been implicated as the cause of LABD, vancomycin being the most frequent ${ }^{6-12}$. LABD can occur anytime from 1 day to 1 month following the initial administration of vancomycin ${ }^{10}$. In addition to the usual vesicul- obullous presentation, vancomycin-induced LABD can also appear as erythematous papules, erosions, urticarial lesions, and eczematous patches ${ }^{9}$. The histological features may be similar to other vesiculobullous diseases, but direct immunofluorescence microscopy commonly demonstrates a characteristic linear band of IgA along the basement membrane zone. Due to the heterogeneous clinical features, LABD must be differentiated from a number of diseases including pemphigus vulgaris, bullous pemphigoid, dermatitis herpetiformis, and erythema multiforme ${ }^{7,9}$.

The etiology of LABD is yet to be fully elucidated, but some have reported the presence of a group of autoantigens that have been found to be targets in the idiopathic form of LABD. Of note, the $97 \mathrm{kD}$ ectodomain of the $180 \mathrm{kD}$ bullous pemphigoid antigen and type-VII collagen are two of the most commonly identified antigens, and may play a role in pathogenesis ${ }^{13}$. In the case of druginduced LABD, there are far fewer cases that have been reported, and thus it has been harder to characterize the target antigen involved. Two studies have found antibodies to the $230 \mathrm{kD}$ antigen, the $97 \mathrm{kD}$ antigen, and type-VII collagen in nonvancomycin drug-induced $\mathrm{LABD}^{14,15}$, and one study reported two patients with vancomycin-induced LABD with autoantibodies against BP180 and LAD $285^{10}$.

The severity of the reaction does not appear to correlate with serum vancomycin levels. In nearly all the reported cases of vancomycin-induced LABD, the bullous eruption resolved after discontinuing vancomycin. In occasional cases, dapsone or prednisolone was used as an alternative to stopping the antibiotic. ${ }^{11}$ However, in case of persisting disease, other etiologic factors must be considered.

Drug-induced LABD shows some different characteristics compared to the idiopathic variant. Recent

Table 1. Summary of the reported cases of vancomycin-induced LABD in Korea

\begin{tabular}{|c|c|c|c|c|c|c|}
\hline No. & Age/Sex & Comorbid disease & Distribution of lesions & Treatment & Time to resolution & Reference \\
\hline 1 & $70 / F$ & Pyogenic arthritis & Trunk, extremities & DC vanc & & 10 \\
\hline 2 & $70 / \mathrm{M}$ & Pyogenic arthritis & $\begin{array}{l}\text { Trunk, genitalia, } \\
\text { lower extremities }\end{array}$ & $\begin{array}{l}\text { DC vancomycin } \\
\text { Dapsone }\end{array}$ & 2 weeks & 11 \\
\hline 3 & $45 / F$ & Urinary tract infection & Trunk, extremities & DC vancomycin & 2 weeks & Our case \\
\hline
\end{tabular}

DC: discontinuation 
reviews suggest that in drug-induced $\mathrm{LABD}$, mucosal or conjunctival lesions are lacking, while up to $40 \%$ of patients with idiopathic LABD have mucosal involvement. Also, in drug-induced LABD, there is remission and clearance of immune deposits upon withdrawal of the drug, whereas only $10 \sim 50 \%$ of patients with idiopathic LABD have spontaneous remission. Reports also show patients with drugnduced LABD tend to be older than patients with idiopathic LABD?

Our patient represents a typical case of vancomycinnduced LABD. She was diagnosed with a persistent urinary tract infection, and was first treated with vancomycin. After 13 days of treatment, pruritic, multiple, tense vesicles with an erythematous base and confluent erythematous papules and plaques suddenly appeared on the trunk, inguinal areas, and upper thighs. Fluconazole was administered after the appearance of the skin manifestations; furthermore, the skin lesions resolved spontaneously after the discontinuation of vancomycin; therefore, vancomycin was considered to be the most etiologic factor. Histological findings showed a subepidermal blister with an inflammatory infiltrate, and direct immunofluorescence revealed a characteristic linear band of $\operatorname{IgA}$. Based on these findings, a diagnosis of vancomycin-nduced LABD was able to be made without much difficulty. Table 1 summarizes the three cases of vancomycin-induced LABD reported to date in Korea including this case. Our case differs in that the age of our patient is relatively younger than those of the previously reported cases, which indicates that old age, though relevant, is not a requisite factor in the pathogenesis of drug-induced LABD.

Vancomycin use has been increasing steadily due to the recent rise in the rate of MRSA infection and it is of even more importance to recognize the symptoms of drug complications. However, it is not always easy to identify the causative drug in LABD, since most patients are on a multi-drug regimen. Therefore, for any patient who presents with an acute outbreak of vesicles centered mainly on the trunk and extremities brought on by the initiation of a certain drug, a diagnosis of linear IgA bullous dermatosis must be considered.

\section{REFERENCES}

1. Rao CL, Hall RP. Linear IgA dermatosis and chronic bullous disease of childhood, In: Freedberg IM, Eisen AZ, Wolff K, Austen KF, Goldsmith LA, Katz SI, editors. Fitzpatrick's dermatology in general medicine. 6th ed. New York: McGraw-Hill, 2003:587-592.

2. Avci O, Okmen M, Cetiner S. Acetaminophennduced linear IgA bullous dermatosis. J Am Acad Dermatol 2003;48:299-300.

3. Bachot N, Wechsler J, Demoule A, Roujeau JC. Amiodarone-related linear IgA bullous dermatosis. J Am Acad Dermatol 200349:E11-12.

4. Cerottini JP, Ricci C, Guggisberg D, Panizzon RG. Drug-induced linear IgA bullous dermatosis probably induced by furosemide. J Am Acad Dermatol 1999;41:103-105.

5. Tran D, Kossard S, Shumack S. Phenytoin-induced linear IgA bullous dermatosis mimicking toxic epidermal necrolysis. Aus J Dermatol 2003;44:284286.

6. Onodera H, Mihm MC Jr, Yoshida A, Akasaka T. Drug-Induced linear IgA bullous dermatosis. J Dermatol 2005;32:759-764.

7. Waldman MA, Black DR, Callen JP. Vancomycininduced linear IgA bullous disease presenting as toxic epidermal necrolysis. Clin Exp Derm 2004; 29:633-636.

8. Jones DH, Todd M, Craig TJ. Early diagnosis is key in vancomycin-induced linear IgA bullous dermatosis and Steven-Johnson's syndrome. J Am Osteopath Assoc 2004;104:157-163.

9. Armstrong AW, Fazeli A, Yeh SW, Mackoll BT, Liu V. Vancomycin-induced linear IgA disease manifesting as bullous erythema multiforme. J Cutan Pathol 2004;31:393-397.

10. Palmer RA, Ogg G, Allen J, Banerjee A, Ryatt KS, Ratnavel R, et al. Vancomycin-induced linear IgA disease with autoantibodies to BP180 and LAD285. Br J Dermatol 2001;145:816-820.

11. Chin HW, Jang HS, Jo JH, Kim MB, Oh CK, Kwon KS. A case of linear IgA bullous dermatosis probably caused by vancomycin. Korean J Dermatol 2005;43:416-418.

12. Doh KS, Kim SH, Choi TS, Jang MS, Suh KS, Kim ST. A case of vancomycin-induced linear IgA dermatosis. Korean J Dermatol 2002;40:1132-1135.

13. Zone JJ, Taylor TB, Meyer LJ, Petersen MJ. The $97 \mathrm{kD}$ linear IgA bullous disease antigen is identical to a portion of the extracellular domain of the $180 \mathrm{kDa}$ bullous pemphigoid antigen, BPAg2. J Invest Dermatol 1998;110:207-210. 
14. Wakelin SH, Allen J, Zhou S, Wojnarowska F. Drug-induced linear IgA disease with antibodies to collagen VII. Br J Dermatol 1998;138:310-314.

15. Paul C, Wolkenstein P, Prost C, Caux F, Rostoker
G, Heller M, et al. Drug-induced linear IgA disease: target antigens are heterogenous. $\mathrm{Br} \mathrm{J}$ Dermatol 1997;136:406-411. 